\title{
ASYMPTOTIC PROPERTIES OF NORMAL AND NONNORMAL HOLOMORPHIC MAPPINGS
}

\author{
BY KYONG T. HAHN ${ }^{1}$
}

Let $\Omega$ be a bounded domain in $\mathbf{C}^{m}$ with Kobayashi metric $k_{\Omega}$ and the corresponding differential metric $K_{\Omega}$. Let $N$ be a hermitian manifold with hermitian inner product $h_{N}$ and hermitian metric $d_{N}$. Let $\mathcal{H}(\Omega, N)$ denote the class of all holomorphic mappings $f: \Omega \rightarrow N$.

In this paper, generalizing the classical notions of normal functions [7], Bloch functions [1], regular sequences [9], and $P$-point sequences [3] of one complex variable to holomorphic mappings in $\mathcal{H}(\Omega, N)$ we obtain certain relations existing between these notions. (These notions have been generalized by several authors. See $[4,10,11]$ for example.) These results are then used to draw some interesting conclusions on the boundary behavior of normal and nonnormal holomorphic mappings.

The context of this paper and other related results with complete proofs will be published elsewhere [5].

We shall say that $f \in \mathcal{H}(\Omega, N)$ is: normal if $\Omega$ is homogeneous, i.e., the group of holomorphic automorphisms Aut $(\Omega)$ is transitive, and the family $\{f \circ \varphi: \varphi \in \operatorname{Aut}(\Omega)\}$ forms a normal family (in the sense of $\mathrm{H}$. Wu [12]); Bloch if

$$
Q f \equiv \sup \{(Q f)(z): z \in \Omega\}<\infty,
$$

where

$$
(Q f)(z)=\sup _{|\xi|=1} \frac{h_{N}(f(z), d f(z) \xi)}{K_{\Omega}(z, \xi)} \quad\left(z \in \Omega, \xi \in \mathbf{C}^{m}\right) .
$$

Denote by $\mathcal{N}(\Omega, N)$ and $B(\Omega, N)$ the classes of normal and Bloch mappings $f: \Omega \rightarrow N$, respectively.

THEOREM 1. Let $\Omega$ be a homogeneous bounded domain and $N$ a complete hermitian manifold. Then $B(\Omega, N)$ is a proper subset of $\mathcal{N}(\Omega, N)$. If, in particular, $N$ is compact, then $B(\Omega, N)=\mathcal{N}(\Omega, N)$. Moreover, if $\Omega$ is symmetric and $N$ is negatively curved, i.e., the holomorphic sectional curvature of $N$ is bounded above by a negative constant, then $B(\Omega, N)=H(\Omega, N)$. In the opposite direction, if $\Omega$ is strongly pseudoconvex and $N=\mathbf{C}^{n}$, then $B\left(\Omega, \mathbf{C}^{n}\right)$ contains all bounded holomorphic mappings properly.

Following Seidel and Walsh [9] and Gauthier [3], we shall define a sequence $\left\{p_{n}\right\}$ of points in $\Omega$ to be: regular for $f \in \mathcal{H}(\Omega, N)$ if there exists a $\delta>0$ such

Received by the editors May 16, 1983 and, in revised form, June 7, 1983.

1980 Mathematics Subject Classification. Primary 32H25, 32A40; Secondary 30D45.

${ }^{1}$ This work was supported in part by NSF MCS80-02915.

(C) 1984 American Mathematical Society $0273-0979 / 84 \$ 1.00+\$ .25$ per page 
that for every $\left\{q_{n}\right\}$ in $\Omega$, with $k_{\Omega}\left(p_{n}, q_{n}\right)<\delta$ for all $n$,

$$
\lim _{n \rightarrow \infty} d_{N}\left(f\left(p_{n}\right), f\left(q_{n}\right)\right)=0 ;
$$

a $P$-point sequence if there exist a sequence $\left\{q_{n}\right\}$ in $\Omega$ and an $\epsilon>0$ such that

$$
\lim _{n \rightarrow \infty} k_{\Omega}\left(p_{n}, q_{n}\right)=0 \text { but } \limsup _{n \rightarrow \infty} d_{N}\left(f\left(p_{n}\right), f\left(q_{n}\right)\right) \geq \epsilon \text {. }
$$

See also $[11]$.

The following statements are easily seen from the definitions and by examples (see [5]).

(a) Any regular sequence of an $f \in \mathcal{H}(\Omega, N)$ cannot be a $P$-point sequence. Therefore, every $P$-point sequence is irregular. The converse is, in general, false.

(b) Mappings in $B(\Omega, N)$ cannot possess a $P$-point sequence in $\Omega$, while mappings in $\mathcal{N}(\Omega, N)$ can for noncompact $N$.

(c) If $\left\{p_{n}\right\}$ is a $P$-point sequence of $f \in \mathcal{N}(\Omega, N)$ then $\left\{f\left(p_{n}\right)\right\}$ is compactly divergent in $N$ in the sense that for each compact $E$ in $N$ there exists an $n_{0}>0$ such that $f\left(p_{n}\right) \notin E$ for $n \geq n_{0}$.

THEOREM 2. Let $\Omega$ be a homogeneous bounded domain in $\mathbf{C}^{m}$ and $N$ compact. Then $f \in \mathcal{N}(\Omega, N)$ if and only if $f$ has no $P$-point sequence in $\Omega$.

Combining Theorems 1 and 2, we obtain

Corollary. Let $\Omega$ and $N$ be given as in Theorem 2 . The following are equivalent for $f \in \mathcal{H}(\Omega, N)$.

(a) $f \notin \mathcal{N}(\Omega, N)$.

(b) $\lim \sup _{z \in \Omega}(Q f)(z)=\infty$.

(c) $f$ has a $P$-point sequence in $\Omega$.

Suppose that $\Omega$ is complete with respect to $k_{\Omega}$. Then a compact exhaustion $\left\{\Omega_{n}\right\}$ can be constructed by $\Omega_{n}=\left\{z \in \Omega: k_{\Omega}\left(z_{0}, z\right) \leq n\right\}, n=1,2, \ldots$, where $z_{0}$ is a fixed point in $\Omega$. The maps $f \in B(\Omega, N)$ for which

$$
\lim _{n \rightarrow \infty}\left[\sup _{z \in \Omega \backslash \Omega_{n}}(Q f)(z)\right]=0
$$

constitute an interesting subclass of $B(\Omega, N)$. This subclass will be denoted by $B_{0}(\Omega, N)$.

THEOREM 3. Let $\Omega$ and $N$ be given as in Theorem 2. Then $f \in B_{0}(\Omega, N)$ if and only if every sequence $\left\{p_{n}\right\}$ in $\Omega$ is regular.

The standard normal family argument, Ascoli theorem [12], and generalized Schwarz lemma [6] are the main tools used in the proofs of Theorems 1-3.

More relationships between nonnormal holomorphic maps and the existence of a $P$-point sequence are established in the following theorems:

THEOREM 4. Let $\Omega$ be a bounded domain in $\mathbf{C}^{m}$ and $N$ any hermitian manifold. Suppose there exist sequences $\left\{p_{n}\right\}$ in $\Omega$ and $\left\{r_{n}\right\}, r_{n}>0$, with $\lim _{n \rightarrow \infty}\left(r_{n} / \delta_{\Omega}\left(p_{n}\right)\right)=0$, where $\delta_{\Omega}(z)$ denotes the distance form $z$ to $\partial \Omega$, such that $\left\{f\left(p_{n}+r_{n} \zeta\right)\right\}, f \in \mathcal{H}(\Omega, N)$, converges locally uniformly to a nonconstant holomorphic mapping $g \in \mathcal{H}\left(\mathbf{C}^{m}, N\right)$. Then $\left\{p_{n}\right\}$ is a $P$-point sequence for $f$. If, in particular, $\Omega$ is homogeneous and $N$ is compact, then $f$ is nonnormal. 
THEOREM 5. Let $\Omega$ be a bounded domain in $\mathbf{C}^{m}$ and $N$ a compact manifold. If $f \in \mathcal{H}(\Omega, N)$ satisfies

$$
\sup _{z \in \Omega} \Lambda_{f}(z) \delta_{\Omega}(z)=\infty,
$$

where $\Lambda_{f}(z)=\sup _{|\xi|=1} h_{N}(f(z), d f(z) \xi)\left(z \in \Omega, \xi \in \mathbf{C}^{m}\right)$, then there exist sequences $\left\{p_{n}\right\}$ in $\Omega$ and $\left\{r_{n}\right\}, r_{n}>0$, with $\lim _{n \rightarrow \infty}\left(r_{n} / \delta_{n}\left(p_{n}\right)\right)=0$ such that $\left\{f\left(p_{n}+r_{n} \zeta\right)\right\}$ converges locally uniformly in $\mathbf{C}^{m}$ to a nonconstant holomorphic mapping $g \in \mathcal{H}\left(\mathbf{C}^{m}, N\right)$.

The proofs of Theorems 4 and 5 follow closely the method of [8].

An honest generalization of Theorem 1 of [8] to more than one complex variable does not hold, as can be shown by an example [5]. This may be due to the fact that the "approach region" along the sequence $\left\{p_{n}\right\}$ in $\Omega$ is much less restricted in higher-dimensional cases. But we can prove the following generalization using the method of [8].

TheOREM 6. Let $D$ be the open unit disc in $\mathbf{C}$ and $N$ any compact manifold. The family $\mathcal{F} \subset \mathcal{H}(D, N)$ is normal if and only if for every choice of sequences $\left\{z_{n}\right\}$ in $D,\left\{f_{n}\right\}, f_{n} \in \mathcal{F}$, and $\left\{r_{n}\right\}, r_{n}>0$, with $\lim _{n \rightarrow \infty} r_{n} /\left(1-\left|z_{n}\right|\right)=0$, the sequence $\left\{f_{n}\left(z_{n}+r_{n} \zeta\right)\right\}$ converges locally uniformly to a constant mapping on $\mathbf{C}$.

From this theorem we have the following criterion for the tautness of a complex submanifold of a compact hermitian manifold.

COROLlaRY 1. Let $X$ be a closed complex submanifold of a compact hermitian manifold $N$. Then $X$ is taut, i.e., $\mathcal{H}(D, X)$ is a normal family if and only if for every choice of sequences $\left\{z_{n}\right\}$ in $D,\left\{f_{n}\right\}$ in $\mathcal{H}(D, X)$ and $\left\{r_{n}\right\}$, $r_{n}>0$, with $\lim _{n \rightarrow \infty} r_{n} /\left(1-\left|z_{n}\right|\right)=0$, the sequence $\left\{f_{n}\left(z_{n}+r_{n} \zeta\right)\right\}$ converges locally uniformly to a constant on $\mathbf{C}$.

In particular, we obtain Brody's result [2].

CoROllary 2. A compact complex manifold $N$ is hyperbolic if and only if there is no nonconstant holomorphic mapping $f: \mathbf{C} \rightarrow N$.

\section{REFERENCES}

1. J. M. Anderson, J. Clunie and Ch. Pommerenke, On Bloch functions and normal functions, J. Reine Angew. Math. 270 (1974), 12-37.

2. R. Brody, Compact manifolds and hyperbolicity, Trans. Amer. Math. Soc. 235 (1978), 213-219.

3. P. Gauthier, A criterion for normalcy, Nagoya Math. J. 32 (1968), 277-282.

4. K. T. Hahn, Holomorphic mappings of the hyperbolic space into the complex Euclidean space and the Bloch theorem, Canad. J. Math. 27 (1975), 446-458.

5. , Asymptotic behavior of normal mappings of several complex variables, Canad. J. Math. (to appear).

6. S. Kobayashi, Hyperbolic manifolds and holomorphic mappings, Dekker, New York, 1970.

7. O. Lehto and V. I. Virtanen, Boundary behavior and normal meromorphic functions, Acta. Math. 97 (1957), 47-63. 
8. A. J. Lohwater and Ch. Pommerenke, On normal meromorphic functions, Ann. Acad. Sci. Fenn. Ser. A I 550 (1973), 1-12.

9. W. Seidel and J. L. Walsh, On the derivatives of functions analytic in the unit circle and their radii of univalence and of p-valence, Trans. Amer. Math. Soc. 52 (1942), 128-216.

10. R. M. Timoney, Bloch functions in several complex variables, Thesis, Univ. of Illinois, 1978.

11. F. Wicker, Basic properties of normal and Bloch mappings, Thesis, Pennsylvania State Univ., 1975.

12. H. H. Wu, Normal families of holomorphic mappings, Acta Math. 119 (1967), 193-233.

Department of Mathematics, The Pennsylvania State University, UniverSITy PARK, PENNSYlVANIA 16802 